\title{
Structural health monitoring using adaptive LMS filters
}

\section{Mostafa Nayyerloo and J. Geoffrey Chase*}

Department of Mechanical Engineering,

University of Canterbury,

Private Bag 4800, Christchurch 8140, New Zealand

Email: mostafa.nayyerloo@pg.canterbury.ac.nz

Email: geoff.chase@canterbury.ac.nz

*Corresponding author

\section{Gregory A. MacRae}

Department of Civil and Natural Resources Engineering, University of Canterbury, Private Bag 4800, Christchurch 8140, New Zealand

Email: gregory.macrae@canterbury.ac.nz

\section{XiaoQi Chen and Christopher Eric Hann}

Department of Mechanical Engineering, University of Canterbury, Private Bag 4800, Christchurch 8140, New Zealand Email: xiaoqi.chen@canterbury.ac.nz Email: chris.hann@ canterbury.ac.nz

\begin{abstract}
A structure's level of damage is determined using a non-linear model-based method utilising a Bouc-Wen hysteretic model. It employs adaptive Least Mean Squares (LMS) filtering theory in real time to identify changes in stiffness due to modelling error damage, as well as plastic and permanent displacements, which are critical to determining ongoing safety and use. The Structural Health Monitoring (SHM) method is validated on a four-story shear structure model undergoing seismic excitation. For the simulated structure, the algorithm identifies stiffness changes to within $10 \%$ of the true value in $0.20 \mathrm{~s}$, and permanent deflection is identified to within $5 \%$ of the actual as-modelled value using noise-free simulation-derived structural responses.
\end{abstract}

Keywords: SHM; structural health monitoring; adaptive filtering; LMS; least mean squares; Bouc-Wen model; damage detection; non-linear structure; computer vision; line scan camera.

Reference to this paper should be made as follows: Nayyerloo, M., Chase, J.G., MacRae, G.A., Chen, X-Q. and Hann, C.E. (201X) 'Structural health monitoring using adaptive LMS filters', Int. J. Computer Applications in Technology, Vol. X, No. Y, pp.xx-xx.

Biographical notes: Mostafa Nayyerloo is studying towards a $\mathrm{PhD}$ in Mechanical Engineering specialising in Mechatronics at the University of Canterbury. He received his BS and MS in Mechanical Engineering from Iran University of Science and Technology and Semnan University in 2002 and 2006, respectively. He worked for three engineering consulting companies specialised in automotive industries for four years as a design engineer prior to joining University of Canterbury in 2007. His research interests include structural health monitoring (structural dynamics/identification/instrumentation) and inspection robots and embedded systems for measurement.

J. Geoffrey Chase received his BS from Case Western Reserve University in 1986 in Mechanical Engineering. His MS and PhD were obtained at Stanford University in 1991 and 1996 respectively in Mechanical and Civil Engineering. He spent six years with General Motors and five years in consulting in Silicon Valley and came to the University of Canterbury in 2000. His research includes biomedical systems/devices, structural dynamics/vibrations, automatic control, MEMS/micro-fabrication, and embedded systems. He has published over 320 refereed journal and conference papers, and is an inventor of over 10 patents. He is currently on the editorial board of three bioengineering journals. 
Gregory A. MacRae is an Associate Professor in the Department of Civil and Natural Resources Engineering, University of Canterbury. He received his $\mathrm{PhD}$ in 1990 from the same university. He has worked in Japan and California, and was an Associate Professor at the University of Washington, Seattle, prior to returning to Canterbury in 2005. His research interests include structural design, steel structures, structural dynamics, loss estimation and structural instrumentation. He is the past chair of the ASCE Seismic Effects committee. He is on the NZ steel structures code review committee and on the World Seismic Safety Initiative board. He is also the NZSEE representative at the International Association of Earthquake Engineering.

XiaoQi Chen is an Associate Professor and Director for Mechatronics Engineering at the University of Canterbury. He was Senior Research Assistant at the University of Durham (1989-1990), Research Fellow at Brunel University (1990-1992) and Senior Scientist in Singapore Institute of Manufacturing Technology (1992-2006). He obtained his BE from South China University of Technology in 1984, MSc from Brunel University, UK, in 1986, and $\mathrm{PhD}$ from the University of Liverpool, UK, in 1989. He received Singapore National Technology Award in 1999. He also received the China-UK Technical Co-Operation Award.

Christopher E. Hann, since 2007, has been a Sir Charles Hercus Health Research Fellow. He worked as a mathematics teaching fellow until 2003, and then joined the Department of Mechanical Engineering in 2004 as a postdoc. He received his BSc and PhD in Mathematics from the University of Canterbury in 1996 and 2001, respectively. His research includes biomedical systems and parameter identification, control systems and computer vision. He has published over 150 refereed journal and conference papers, and is an inventor of several patents. He is currently on the editorial board for a bioengineering journal.

\section{Introduction}

Structural Health Monitoring (SHM) is the process of comparing the current state of a structure's condition relative to a baseline state to detect existence, location and degree of likely damage, particularly after a damaging input (Doherty, 1987). SHM can simplify typical procedures of visual or localised experimental methods, as it does not require visual inspection of the structure. It thus provides valuable data for post-event safety assessments to help optimise recovery planning.

Many current vibration-based SHM methods are based on the idea that changes in modal parameters - frequencies, mode shapes and modal damping - are a result of changes in the physical mass, damping and stiffness properties of the structure (Doebling et al., 1996). These modal methods are typically more applicable to steel-frame and bridge structures where vibration response is highly linear (Doebling et al., 1996; Chase et al., 2004). Wavelet approaches offer a similar approach determining the time at which damage occurred.

A major drawback of all these approaches is their inability to be implemented in real time, on a sample-to-sample basis as the event occurs. Further, their reliance on modal properties has potential problems. The modal properties have been shown in some cases to be non-robust in the presence of strong noise and insensitive to small amounts of damage (Hou et al., 2000).

Adaptive fading Kalman filter (Loh et al., 2000) and adaptive $\mathrm{H}_{\infty}$ filter techniques (Sato and Qi, 1998) to achieve real-time capable, or near real-time capable results, provide identification of modal parameters in real time that comes with significant computational cost and complexity. Moreover, like other linear approaches they are not applicable to the typical non-linearities found in seismic structural responses.
In contrast, direct identification of changes in stiffness and/or plastic and permanent deflections would offer the postearthquake outputs desired by engineers. The goal is to obtain these stiffness changes in real time in a computationally efficient and robust fashion. Model-based methods combined with modern filtering theory offer that opportunity.

Least Mean Squares (LMS)-based SHM has been used for a benchmark problem (Chase et al., 2004), and also for a non-linear rocking structure (Chase et al., 2005), to directly identify changes in structural stiffness only. They are robust with fast convergence and low computational cost. However, they do not identify plastic and permanent deflections and require full state structural response measurement.

This preliminary research shows that the modified LMSbased SHM algorithm proposed for non-linear yielding structures offers real-time identification of changes in stiffness plus plastic deflections using noise-free structural responses. The noise effect will be taken into account at later stages.

Due to a variety of practical constraints, direct high frequency measurement of displacement and velocity is not typically possible. Displacement and velocity are often estimated by integration of measured acceleration and are subject to drift and error. However, this error can be corrected using low frequency displacement data obtained via a variety of sensors, such as ground-based GPS or fibre optics. The work described below is predicated on the idea that emerging high-speed line scan cameras can offer a robust and high-speed displacement measure required for the modified LMS-based SHM algorithm proposed for nonlinear yielding structures undergoing seismic excitation. 


\section{Definition of the SHM Problem}

A seismically excited non-linear structure can be modelled at each time step using the incremental equations of motion:

$$
\mathbf{M} \cdot\{\Delta \ddot{v}\}+\mathbf{C} \cdot\{\Delta \dot{v}\}+\mathbf{K}_{\mathbf{T}}(\mathbf{t}) \cdot\{\Delta v\}=-\underline{\mathbf{M}} \cdot \Delta \ddot{x}_{g}
$$

where $\mathbf{M}, \mathbf{C}$ and $\mathbf{K}_{\mathbf{T}}$ are the mass, damping and tangent stiffness matrices of the model, respectively; $\{\Delta v\},\{\Delta \dot{v}\}$ and $\{\Delta \ddot{v}\}$ are the changes in displacement, velocity and acceleration vectors, respectively; and $\Delta \ddot{x}_{g}$ is the change in the ground motion acceleration over the time step. The tangent stiffness matrix of a hysteretic structure can be represented using Bouc-Wen model. For instance, the tangent stiffness matrix of a 4-DOF (four-degree-of-freedom) four-storey shear-type structure, as an example for the tangent stiffness matrix of a hysteretic structure in multidegree-of-freedom case, can be written as:

$$
\begin{aligned}
& \left(\mathbf{K}_{\mathbf{T}}\right)_{11}=\left[\alpha_{1}+\left(1-\alpha_{1}\right) Y_{1} \frac{\Delta z_{1}}{\Delta v_{1}}\right]\left(k_{0}\right)_{1}+\alpha_{2}\left(k_{0}\right)_{2} \\
& \left(\mathbf{K}_{\mathbf{T}}\right)_{12}=-\left[\alpha_{2}+\left(1-\alpha_{2}\right) Y_{2} \frac{\Delta z_{2}}{\Delta v_{2}}\right]\left(k_{0}\right)_{2} \\
& \left(\mathbf{K}_{\mathbf{T}}\right)_{21}=-\alpha_{2}\left(k_{0}\right)_{2} \\
& \left(\mathbf{K}_{\mathbf{T}}\right)_{22}=\left[\alpha_{2}+\left(1-\alpha_{2}\right) Y_{2} \frac{\Delta z_{2}}{\Delta v_{2}}\right]\left(k_{0}\right)_{2}+\alpha_{3}\left(k_{0}\right)_{3} \\
& \left(\mathbf{K}_{\mathbf{T}}\right)_{23}=-\left[\alpha_{3}+\left(1-\alpha_{3}\right) Y_{3} \frac{\Delta z_{3}}{\Delta v_{3}}\right]\left(k_{0}\right)_{3} \\
& \left(\mathbf{K}_{\mathbf{T}}\right)_{32}=-\alpha_{3}\left(k_{0}\right)_{3} \\
& \left(\mathbf{K}_{\mathbf{T}}\right)_{33}=\left[\alpha_{3}+\left(1-\alpha_{3}\right) Y_{3} \frac{\Delta z_{3}}{\Delta v_{3}}\right]\left(k_{0}\right)_{3}+\alpha_{4}\left(k_{0}\right)_{4} \\
& \left(\mathbf{K}_{\mathbf{T}}\right)_{34}=-\left[\alpha_{4}+\left(1-\alpha_{4}\right) Y_{4} \frac{\Delta z_{4}}{\Delta v_{4}}\right]\left(k_{0}\right)_{4} \\
& \left(\mathbf{K}_{\mathbf{T}}\right)_{43}=-\alpha_{4}\left(k_{0}\right)_{4} \\
& \left(\mathbf{K}_{\mathbf{T}}\right)_{44}=\left[\alpha_{4}+\left(1-\alpha_{4}\right) Y_{4} \frac{\Delta z_{4}}{\Delta v_{4}}\right]\left(k_{0}\right)_{4} \\
& \left(\mathbf{K}_{\mathbf{T}}\right)_{13}=\left(\mathbf{K}_{\mathbf{T}}\right)_{14}=\left(\mathbf{K}_{\mathbf{T}}\right)_{24}=\left(\mathbf{K}_{\mathbf{T}}\right)_{31}=\left(\mathbf{K}_{\mathbf{T}}\right)_{41}=\left(\mathbf{K}_{\mathbf{T}}\right)_{42}=0
\end{aligned}
$$

where $\alpha_{i}, i=1, \ldots, 4$, is the $i$ th storey bi-linear factor, which determines the change in slope between elastic and plastic regimes of that storey, and $z_{i}, i=1, \ldots, 4$, is the dimensionless hysteretic component of the $i$ th storey and is governed by the following first order non-linear differential equation (Constantinou and Tadjbakhsh, 1985):

$$
\dot{z}_{i}(t)=\frac{A_{i} \dot{r}_{i}(t)-\beta_{i}\left|\dot{r}_{i}(t) \| z_{i}\right|^{n_{i}-1} z_{i}-\gamma_{i} \dot{r}_{i}(t)\left|z_{i}\right|^{n_{i}}}{Y_{i}}, i=1, \ldots, N
$$

where $\mathrm{A}_{i}$ (usually 1.0 ), $\beta_{i}$ (0.1 to 0.9$), \gamma_{i}\left(-0.9\right.$ to 0.9 ) and $n_{i}$ (1 to 3 , usually 1$)$ are stiffness, loop fatness, loop pinching and abruptness parameters in a classical Bouc-Wen model, respectively. Further, $n_{i}$, the power factor, determines the curve from elastic to plastic force-deflection behaviour of each storey. $\dot{r}_{i}(t)$ is the velocity of storey $i$ relative to storey $i-1, Y_{i}$ is the yield displacement of $i$ th story, and $N$ is the number of stories. The five dimensionless parameters, $A_{i}, \beta_{i}$, $\gamma_{i}, n_{i}$ and $\alpha_{i}$ determine the hysteresis loops shape. Neither degradation nor pinching of hysteresis is accounted for by the classical Bouc-Wen model. Over the years, this classical model has been modified to accommodate changes in hysteresis loops arising from deteriorating systems, and the contemporary model can be found in the studies of Baber and Noori (1986). In this study, the classical Bouc-Wen model has been used, and only non-linearities arising from the hysteresis behaviour of the building has been considered.

If damage occurs in the structure from an earthquake, or any other source of damaging excitation, structural properties, such as natural frequency and stiffness may also change, and may be time-varying. For the damaged structure, the equations of motion can be redefined as:

$$
\mathbf{M} \cdot\{\Delta \ddot{\bar{v}}\}+\mathbf{C} \cdot\{\Delta \dot{\bar{v}}\}+\left(\overline{\mathbf{K}}_{\mathbf{T}}+\Delta \overline{\mathbf{K}}_{\mathbf{T}}\right) \cdot\{\Delta \overline{\bar{v}}\}=-\underline{\mathbf{M}} \cdot \Delta \ddot{x}_{g}
$$

where $\{\Delta \ddot{\bar{v}}\},\{\Delta \dot{\bar{v}}\}$ and $\{\Delta \bar{v}\}$ are the measured changes in responses of the damaged structure, $\overline{\mathbf{K}}_{\mathrm{T}}$ is the tangent stiffness matrix of the damaged structure from equation (2) using damaged structural responses, and $\Delta \overline{\mathbf{K}}_{\mathrm{T}}$ contains changes in the tangent stiffness of the structure due to modelling or construction error damage and can be a function of time. Using equation (2), $\Delta \overline{\mathbf{K}}_{\mathrm{T}}$ due to modelling or construction damage can be written as:

$$
\begin{aligned}
& \left(\Delta \mathbf{K}_{\mathbf{T}}\right)_{11}=\left[\alpha_{1}+\left(1-\alpha_{1}\right) Y_{1} \frac{\Delta z_{1}}{\Delta v_{1}}\right]\left(\Delta k_{0}\right)_{1}+\alpha_{2}\left(\Delta k_{0}\right)_{2} \\
& \left(\Delta \mathbf{K}_{\mathbf{T}}\right)_{12}=-\left[\alpha_{2}+\left(1-\alpha_{2}\right) Y_{2} \frac{\Delta z_{2}}{\Delta v_{2}}\right]\left(\Delta k_{0}\right)_{2} \\
& \left(\Delta \mathbf{K}_{\mathbf{T}}\right)_{21}=-\alpha_{2}\left(\Delta k_{0}\right)_{2} \\
& \left(\Delta \mathbf{K}_{\mathbf{T}}\right)_{22}=\left[\alpha_{2}+\left(1-\alpha_{2}\right) Y_{2} \frac{\Delta z_{2}}{\Delta v_{2}}\right]\left(\Delta k_{0}\right)_{2}+\alpha_{3}\left(\Delta k_{0}\right)_{3} \\
& \left(\Delta \mathbf{K}_{\mathbf{T}}\right)_{23}=-\left[\alpha_{3}+\left(1-\alpha_{3}\right) Y_{3} \frac{\Delta z_{3}}{\Delta v_{3}}\right]\left(\Delta k_{0}\right)_{3} \\
& \left(\Delta \mathbf{K}_{\mathbf{T}}\right)_{32}=-\alpha_{3}\left(\Delta k_{0}\right)_{3} \\
& \left(\Delta \mathbf{K}_{\mathbf{T}}\right)_{33}=\left[\alpha_{3}+\left(1-\alpha_{3}\right) Y_{3} \frac{\Delta z_{3}}{\Delta v_{3}}\right]\left(\Delta k_{0}\right)_{3}+\alpha_{4}\left(\Delta k_{0}\right)_{4} \\
& \left(\Delta \mathbf{K}_{\mathbf{T}}\right)_{34}=-\left[\alpha_{4}+\left(1-\alpha_{4}\right) Y_{4} \frac{\Delta z_{4}}{\Delta v_{4}}\right]\left(\Delta k_{0}\right)_{4} \\
& \left(\Delta \mathbf{K}_{\mathbf{T}}\right)_{43}=-\alpha_{4}\left(\Delta k_{0}\right)_{4}
\end{aligned}
$$




$$
\begin{aligned}
\left(\Delta \mathbf{K}_{\mathbf{T}}\right)_{44} & =\left[\alpha_{4}+\left(1-\alpha_{4}\right) Y_{4} \frac{\Delta z_{4}}{\Delta v_{4}}\right]\left(\Delta k_{0}\right)_{4} \\
\left(\Delta \mathbf{K}_{\mathbf{T}}\right)_{13} & =\left(\Delta \mathbf{K}_{\mathbf{T}}\right)_{14}=\left(\Delta \mathbf{K}_{\mathbf{T}}\right)_{24}=\left(\Delta \mathbf{K}_{\mathbf{T}}\right)_{31} \\
& =\left(\Delta \mathbf{K}_{\mathbf{T}}\right)_{41}=\left(\Delta \mathbf{K}_{\mathbf{T}}\right)_{42}=0
\end{aligned}
$$

Identifying the $\Delta \overline{\mathbf{K}}_{\mathrm{T}}$ term enables the structure's condition to be directly monitored without using modal parameters.

To determine $\Delta \overline{\mathbf{K}}_{\mathrm{T}}$ using adaptive LMS, following the method proposed by Chase et al. (2004), a new form of $\Delta \overline{\mathbf{K}}_{\mathrm{T}}$ is defined with time-varying scalar parameters $\hat{\alpha}_{i}$, to be identified using the LMS filter. For instance, $\Delta \overline{\mathbf{K}}_{\mathrm{T}}$ for a 4-DOF four-story shear building is sub-divided into four matrices to allow independent identification of changes in linear elastic stiffness of each story, i.e. $\left(\Delta k_{0}\right)_{1},\left(\Delta k_{0}\right)_{2},\left(\Delta k_{0}\right)_{3}$ and $\left(\Delta k_{0}\right)_{4}$ :

$$
\Delta \overline{\mathbf{K}}_{\mathbf{T}}=\hat{\alpha}_{1} \mathbf{K}_{1}+\hat{\alpha}_{2} \mathbf{K}_{2}+\hat{\alpha}_{3} \mathbf{K}_{3}+\hat{\alpha}_{4} \mathbf{K}_{4}
$$

where

$$
\begin{aligned}
\mathbf{K}_{1} & =\left[\begin{array}{cccc}
\alpha_{1}+\left(1-\alpha_{1}\right) Y_{1} \frac{\Delta \bar{z}_{1}}{\Delta \bar{v}_{1}} & 0 & 0 & 0 \\
0 & 0 & 0 & 0 \\
0 & 0 & 0 & 0 \\
0 & 0 & 0 & 0
\end{array}\right] \\
\mathbf{K}_{2} & =\left[\begin{array}{cccc}
\alpha_{2} & -\left[\alpha_{2}\left(1-\alpha_{2}\right) Y_{2} \frac{\Delta \bar{z}_{2}}{\Delta \bar{v}_{2}}\right] & 0 & 0 \\
-\alpha_{2} & \alpha_{2}+\left(1-\alpha_{2}\right) Y_{2} \frac{\Delta \bar{z}_{2}}{\Delta \bar{v}_{2}} & 0 & 0 \\
0 & 0 & 0 & 0 \\
0 & 0 & 0 & 0
\end{array}\right] \\
\mathbf{K}_{3} & =\left[\begin{array}{cccc}
0 & 0 & 0 & 0 \\
0 & \alpha_{3} & -\left[\alpha_{3}+\left(1-\alpha_{3}\right) Y_{3} \frac{\Delta \bar{z}_{3}}{\Delta \bar{v}_{3}}\right. & 0 \\
0 & -\alpha_{3} & \alpha_{3}+\left(1-\alpha_{3}\right) Y_{3} \frac{\Delta \bar{z}_{3}}{\Delta \bar{v}_{3}} & 0 \\
0 & 0 & 0 & 0
\end{array}\right] \\
\mathbf{K}_{4} & =\left[\begin{array}{cccc}
0 & 0 & 0 & 0 \\
0 & 0 & 0 & 0 \\
0 & \alpha_{4} & -\left[\alpha_{4}+\left(1-\alpha_{4}\right) Y_{4} \frac{\Delta \bar{z}_{4}}{\Delta \bar{v}_{4}}\right. & 0 \\
0 & -\alpha_{4} & \alpha_{4}+\left(1-\alpha_{4}\right) Y_{4} \frac{\Delta \bar{z}_{4}}{\Delta \bar{v}_{4}} & 0
\end{array}\right]
\end{aligned}
$$

and

$$
\hat{\alpha}_{1}=\left(\Delta k_{0}\right)_{1}, \hat{\alpha}_{2}=\left(\Delta k_{0}\right)_{2}, \hat{\alpha}_{3}=\left(\Delta k_{0}\right)_{3}, \hat{\alpha}_{4}=\left(\Delta k_{0}\right)_{4} .
$$

Hence equation (6) can be expressed as:

$$
\Delta \overline{\mathbf{K}}_{\mathbf{T}}=\sum_{i=1}^{n} \hat{\alpha}_{i} \mathbf{K}_{\mathbf{i}}
$$

where $n$ is the number of degrees of freedom of the model, and $\mathbf{K}_{\mathbf{i}}$ is the corresponding time-varying matrix to $i$ th DOF in equations (6)-(10). Rewriting equation (4) using equations (6)-(12) yields:

$$
\sum_{i=1}^{n} \hat{\alpha}_{1} \mathbf{K}_{\mathbf{i}} \cdot\{\Delta \bar{v}\}=-\underline{\mathbf{M}} \cdot \Delta \ddot{x}_{\varepsilon}-\mathbf{M} \cdot\{\Delta \Delta \ddot{\bar{v}}\}-\mathbf{C} \cdot\{\Delta \dot{\bar{v}}\}-\overline{\mathbf{K}}_{\mathbf{T}} \cdot\{\Delta \overline{\bar{v}}\}
$$

In equation (13), changes in responses of the damaged structure $\{\Delta \ddot{\bar{v}}\},\{\Delta \dot{\bar{v}}\}$ and $\{\Delta \bar{v}\}$ are measured, and the matrix $\overline{\mathbf{K}}_{\mathrm{T}}$ at each time step is calculated using equations (2) and (3). The $Y_{i} \Delta \bar{z}_{i}$ term in $\overline{\mathbf{K}}_{\mathbf{T}}$ and $\mathbf{K}_{\mathbf{i}}$ matrices can be redefined by introducing a hysteretic displacement, $h_{i}$ for each storey as:

$$
h_{i}=Y_{i} z_{i}, \quad i=1, \ldots, N
$$

where $Y_{i}$ and $z_{i}$ are the yield displacement and the hysteretic component of the $i$ th storey, respectively. Assuming $A_{i}=1, \beta_{i}=0.5$ and $\gamma_{i}=0.5$ in equation (3), $\dot{h}_{i}(t)$ can be written as:

$$
\dot{h}_{i}(t)=\dot{r}_{i}(t)\left\{1-0.5\left[1+\operatorname{sign}\left(\dot{r}_{i}(t) h_{i}(t)\right)\right]\left|\frac{h_{i}(t)}{Y_{i}}\right|^{n_{i}}\right\}, i=1, \ldots, N
$$

where $\dot{r}_{i}(t)$ is the velocity of storey $i$ relative to storey $i-1$, $Y_{i}$ is the yield displacement of $i$ th story, $n_{i}$ is the power factor and $N$ is the number of stories. Using equation (15) and assuming constant $\dot{h}_{i}$ at each time step, $Y_{i} \Delta z_{i}$ or $\Delta h_{i}$, changes in hysteretic displacement of storey $i$ over each time step can be calculated as:

$$
\begin{gathered}
h_{i}(t+\Delta t)-h_{i}(t)=\dot{r}_{i}(t)\left\{1-0.5\left[1+\operatorname{sign}\left(\dot{r}_{i}(t) h_{i}(t)\right)\right]\left|\frac{h_{i}(t)}{Y_{i}}\right|^{n_{i}}\right\} \Delta t, \\
i=1, \ldots, N
\end{gathered}
$$

where $\Delta t$ is the time step. Thus, $Y_{i} \Delta \bar{\Delta}_{i}$ or $\Delta \bar{h}_{i}$, changes in damaged hysteretic displacement of $i$ th storey over each time step, can be determined from equation (16) using damaged structural responses.

In this fashion, plastic displacement of storey $i$ in the Bouc-Wen model, $D_{i}(t)$, is defined as:

$$
D_{i}(t)=\frac{r_{i}(t)-h_{i}(t)}{1+\left(\frac{\alpha_{i}}{1-\alpha_{i}}\right)}, i=1, \ldots, N
$$

where $r_{i}(t)$ is the relative displacement between storey $i$ and storey $i-1, h_{i}$ is the hysteretic displacement and $\alpha_{i}$ is the bilinear factor of $i$ th story.

The damaged structure stiffness, or the effective stiffness changes due to non-linear behaviour such as hysteresis, can then be determined by identifying the $\hat{\alpha}_{i}$ in equation (13) at every discrete time step using equation (18).

$$
\begin{aligned}
\sum_{i=1}^{n} \hat{\alpha}_{i} \mathbf{K}_{\mathbf{i}} \cdot\{\Delta \bar{v}\}_{k}= & -\mathbf{M} \cdot\left(\Delta \ddot{x_{g}}\right)_{k}-\mathbf{M} \cdot\{\Delta \ddot{\bar{v}}\}_{k} \\
& -\mathbf{C} \cdot\{\Delta \dot{\bar{v}}\}_{k}-\overline{\mathbf{K}}_{\mathbf{T}} \cdot\{\Delta \bar{v}\}_{k} \\
= & \{y\}_{k}
\end{aligned}
$$


where $\left(\Delta \ddot{x}_{g}\right)_{k}$ is the change in the input ground acceleration over a given time step of $k$, and $\{\Delta \ddot{\bar{v}}\}_{k},\{\Delta \dot{\bar{v}}\}_{k}$ and $\{\Delta \bar{v}\}_{k}$ are the measured changes in the acceleration, velocity and displacement vectors of the damaged structure over the same time step, respectively. Matrices of $\overline{\mathbf{K}}_{\mathbf{T}}$ and $\mathbf{K}_{\mathbf{i}}$ are calculated sample to sample using equations (2) and (6) with the measured damaged structural responses. The elements of the vector signal $\{y\}_{k}$ can be readily modelled in real time using an adaptive LMS filter so that the coefficients $\hat{\alpha}_{i}$, changes in linear elastic stiffness of each storey due to modelling or construction damage, can be readily determined.

\section{Adaptive LMS filtering}

Adaptive filters are digital filters with coefficients that can change over time. The general idea is to update filter coefficients and assess how well the existing coefficients are performing in modelling a noisy signal, and then adapt the coefficient values to improve performance. The LMS algorithm is one of the most widely used of all the adaptive filtering algorithms and is relatively simple to implement. It is an approximation of the Steepest Descent Method using an estimator of the gradient instead of its actual value, considerably simplifying the calculations and to be readily performed in real-time applications. The goal in this case is to model the individual, scalar elements of the signal $\{y\} k$ of equation (18) using the adaptive LMS filter.

In adaptive LMS filtering, the coefficients are adjusted from sample to sample to minimise the Mean Square Error (MSE), between a measured scalar signal and its modelled value from the filter.

$$
e_{k}=y_{k}-W_{k}^{T} X_{k}=y_{k}-\sum_{i=0}^{m-1} w_{k}(i) x_{k-i}
$$

where $W_{k}$ is the adjustable filter coefficient vector or weight vector at time $k, y_{k}$ is the measured scalar signal at time $k$ to be modelled or approximated, $X_{k}$ is the input vector to the filter, model of current and previous filter inputs, $x_{k-i}$, so $W_{k}^{T} X_{k}$ is the vector dot product output from the filter at time $k$ to model a scalar signal $y_{k}$, and $m$ is the number of prior time steps or taps considered. The Widrow-Hopf LMS algorithm for updating the weights to minimise the error, $e_{k}$, is defined as (Ifeachor and Jervis, 1993):

$$
W_{k+1}=W_{k}+2 \mu e_{k} X_{k}
$$

where $\mu$ is a positive scalar, called step size, that controls the stability and rate of convergence.

To identify $\Delta \overline{\mathbf{K}}_{\mathrm{T}}$ at time $k$, using LMS adaptive filters, we will follow the One-Step method (Chase et al., 2004) and rewrite equation (19) in matrix form by substituting $W_{k}^{T} X_{k}$ with its equivalent from equation (18):

$$
\{e\}_{k}=\{y\}_{k}-\sum_{j=0}^{m-1} \sum_{i=1}^{n} \hat{\alpha}_{i j} \mathbf{K}_{\mathbf{i}} \cdot\{\Delta \bar{v}\}_{k}
$$

Minimising the mean square error with respect to $\hat{\alpha}_{i j}$ using equation (20) yields the following weight update formula for each coefficient in the weight matrix of the SHM problem:

$$
w_{k+1}=w_{k}+2 \mu\{e\}_{k}^{T} \mathbf{K}_{\mathbf{i}} \cdot\{\Delta \bar{v}\}_{k-j}
$$

Summing $\hat{\alpha}_{i j}$ over $j$, yields the $\hat{\alpha}_{i}$, changes in stiffness of each story in equation (18).

\section{Inputs to the SHM problem}

Inputs to this SHM problem are acceleration, velocity and displacement of the structure. Acceleration can be easily measured with low-cost accelerometers at high sampling rates, but due to practical constraints direct high-speed measurement of displacement and velocity is not typically possible. A highspeed displacement sensor would provide displacement, and could be used to derive velocity at low added computational cost. Estimating the velocity using both acceleration and displacement data would provide a more precise estimation of the velocity. To measure displacement of a real structure at high rates, this paper proposes but does not explore the method proposed by Lim and Lim (2008). Using only one high-speed line scan camera and a special pattern explained by Lim and Lim (2008), multiple displacements and motions can be determined in real time at rates of up to tens of $\mathrm{kHz}$. This is more than sufficient for the structural seismic monitoring problem.

\section{Simulated structure}

The algorithm was tested using simulated input data in order to provide proof of concept and quantify the accuracy of the identified parameters: changes in linear elastic stiffness and plastic/permanent displacements of each storey. MATLAB ${ }^{\circledR}$ was used to simulate the responses of the structure shown in Figure 1 using Newmark- $\beta$ integration method. Each storey in this building has a pre-yield stiffness of $1600 \mathrm{kN} / \mathrm{m}$ and mass of 10 tonnes, resulting in an undamped fundamental natural period of $0.45 \mathrm{~s}$ for the structure. A diagonal mass matrix was used in simulation.

Figure1 Simulated 4-DOF four-story shear building

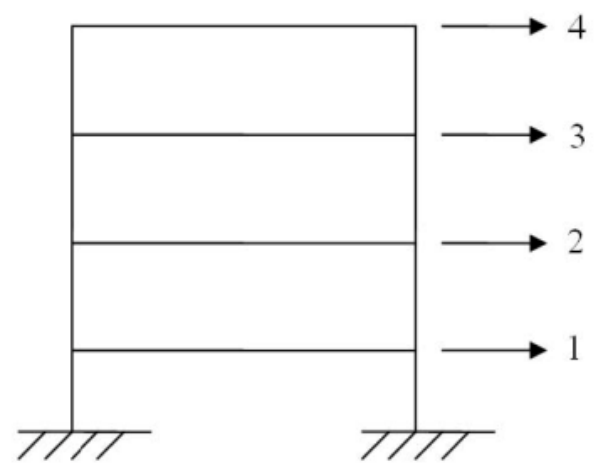


The following damping matrix was constructed for the simulated structure using Caughey method assuming 5\% damping in each mode:

$$
\mathbf{C}=10^{2} \times\left[\begin{array}{cccc}
3.2 & -1.6 & 0 & 0 \\
-1.6 & 3.2 & -1.6 & 0 \\
0 & -1.6 & 3.2 & -1.6 \\
0 & 0 & -1.6 & 1.6
\end{array}\right][\mathrm{kN} . \mathrm{s} / \mathrm{m}]
$$

Each storey was given a yield displacement, $Y$, of $0.02 \mathrm{~m}$, shaping parameter, $n$, of 2 , and a bilinear factor, $\alpha=0.1$. These parameters were chosen to provide realistic nonlinear structural behaviour.

The simulated structure was subjected to the EI Centro earthquake record with Peak Ground Acceleration (PGA) of $0.22 \mathrm{~g}$, with a $10 \%$ reduction in pre-yield stiffness applied to the bottom story at the $10 \mathrm{~s}$ mark. Data was recorded at $500 \mathrm{~Hz}$.

\section{Results}

Typical responses of the bottom story of the simulated fourstory shear building under the EI Centro earthquake are shown in Figure 2. Simulated responses of the structure in damaging event have been used to identify changes in structural stiffness using the adaptive LMS method. As shown in Figures 3, in a worst-case sudden failure situation, the changes in linear elastic stiffness converge to $10 \%$ of the actual value within less than $0.20 \mathrm{~s}$ using 10 taps at $500 \mathrm{~Hz}$ sampling rate.

Figure 2 Responses of the bottom story of the simulated structure subject to the EI Centro earthquake and $10 \%$ sudden failure in the bottom story
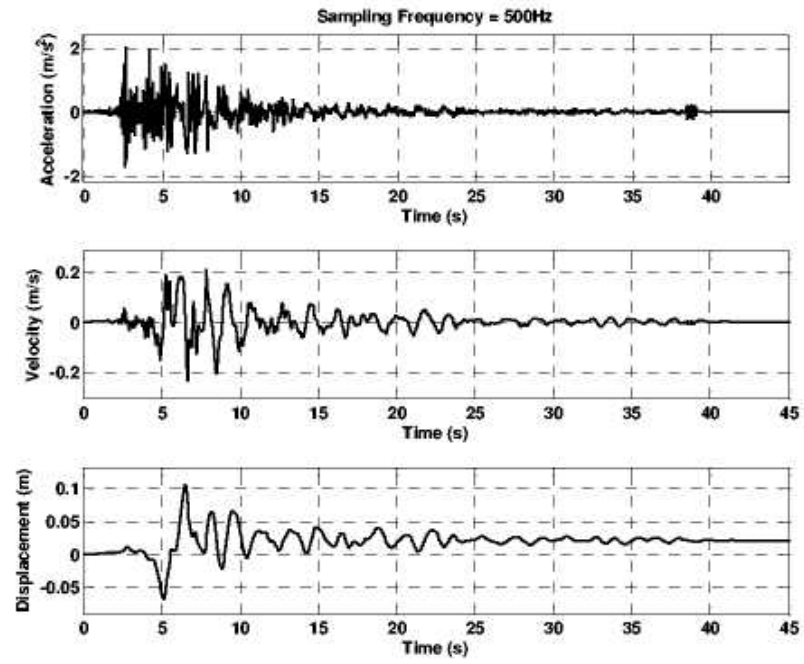

Figures 4 and 5 show that filter approaches faster to the final values of the pre-yield stiffness changes after damage when a higher sampling rate or a greater tap number is used to identify the stiffness changes.
Figure 3 Identified changes in pre-yield stiffness of the bottom story with $10 \%$ sudden failure using adaptive LMS algorithm

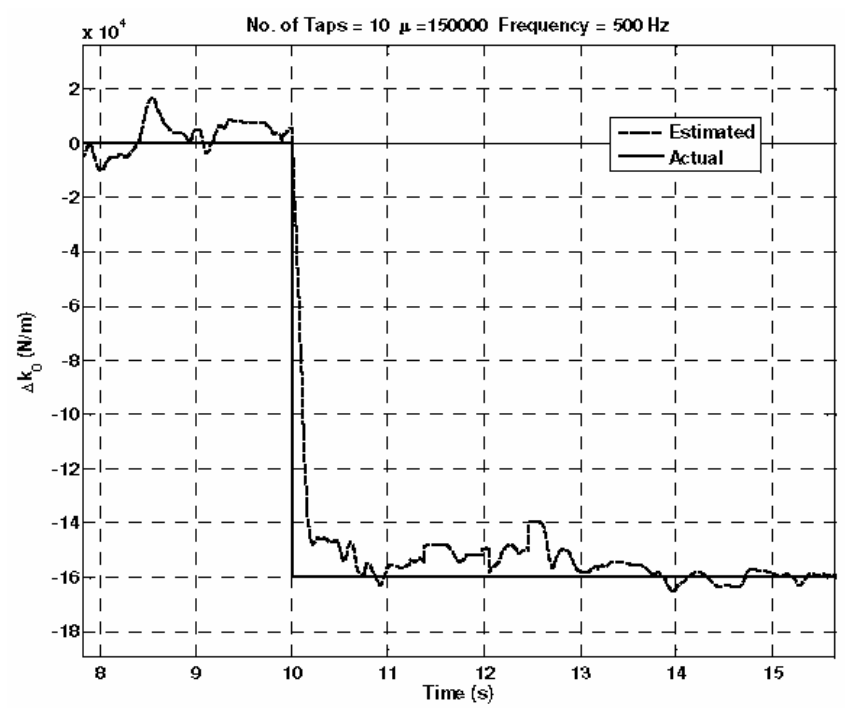

Running the simulation with estimated values for changes in pre-yield stiffness of the structure to obtain identified responses of the damaged structure using Newmark- $\beta$ integration method and equation (16), and then using equation (17) to get the plastic and permanent deflections of the structure, yields Figure 6 . This figure clearly shows that as the filter approaches its final value for changes in stiffness $\left(\Delta \mathrm{k}_{0}\right)$, the plastic deflection approaches its actual value and the error between actual and estimated values for plastic deflections becomes smaller. Over the entire record, the ratio between norms of the error signal in estimating the plastic deflections of the bottom storey and the actual plastic deflection signal is less than $5 \%$. Moreover, the permanent deflection of the bottom storey is identified within less than $5 \%$ of the actual value.

Figure 4 Identified changes in pre-yield stiffness of the bottom story with $10 \%$ sudden failure using adaptive LMS algorithm at different sampling rates

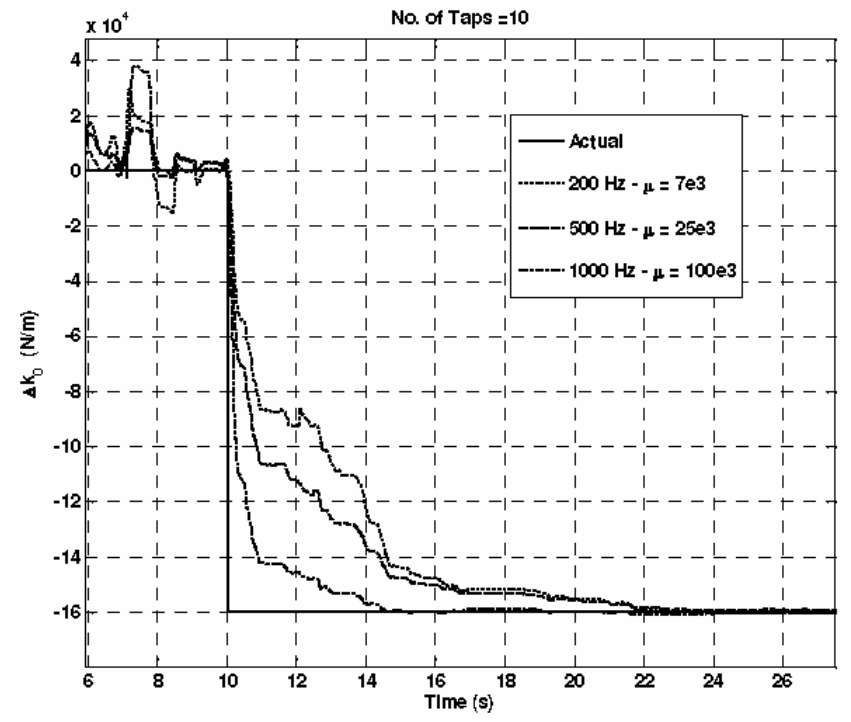


Figure 5 Identified changes in pre-yield stiffness of the bottom story with $10 \%$ sudden failure using adaptive LMS algorithm with different tap numbers

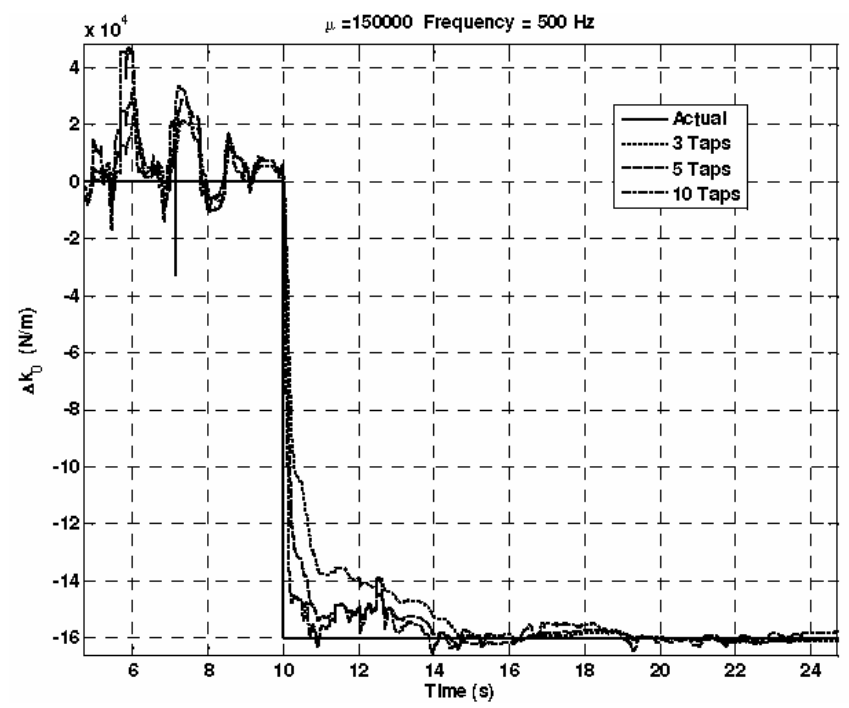

Figure 6 Identified plastic displacements of the bottom story with $10 \%$ sudden failure using estimated changes in pre-yield stiffness of the structure
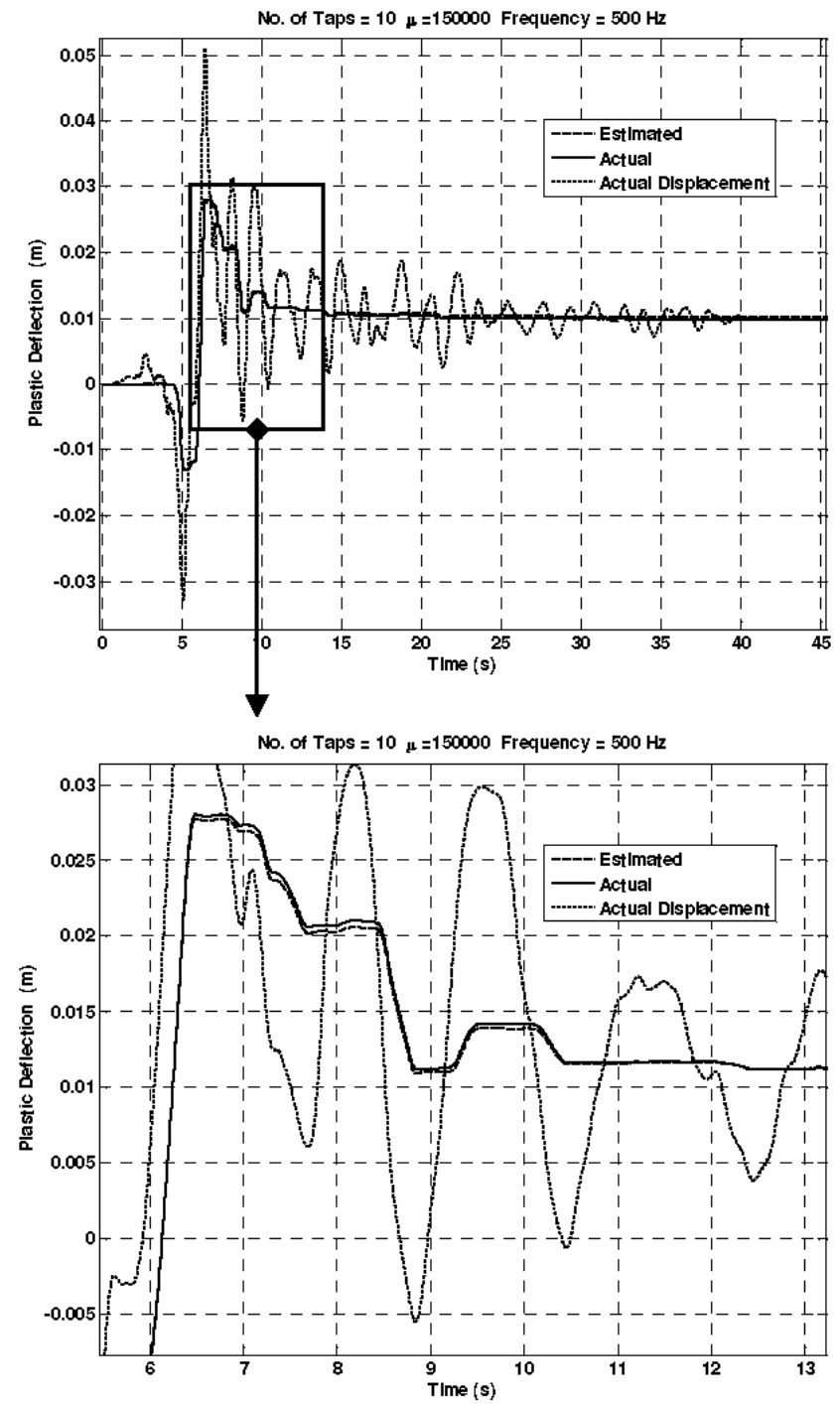

\section{Conclusion}

The developed LMS-based SHM method with a baseline non-linear Bouc-Wen structural model can directly identify plastic deflections and changes in stiffness (modelling or construction error) in real time. The simulation results show that the algorithm identifies stiffness changes to within $10 \%$ of true value in less than $0.20 \mathrm{~s}$, and permanent deflection is identified to within $5 \%$ of actual value using noise-free structural responses. Moreover, over the entire record, norm of the error signal in identifying the plastic deflections over the actual plastic deflection signal is less than $5 \%$. The proposed filter-based identification approach to SHM problems in comparison with existing adaptive methods makes plastic and permanent deflections' identification possible, which is critical for determining ongoing safety of the structure.

\section{References}

Baber, T.T. and Noori, M.N. (1986) 'Modelling general hysteresis behaviour and random vibration application', Journal of Vibration, Acoustics, Stress, and Reliability in Design, Vol. 108, pp.411-420.

Chase, J.G., Hwang, K.L., Barroso, L.R. and Mander, J.B. (2004) 'A simple LMS-based approach to the structural health monitoring benchmark problem', Journal of Earthquake Engineering and Structural Dynamics, Vol. 34, No. 6, pp.575-594.

Chase, J.G., Spieth, H.A., Blome, C.F. and Mander, J.B. (2005) 'LMS-based structural health monitoring of a non-linear rocking structure', Journal of Earthquake Engineering and Structural Dynamics, Vol. 34, pp.909-930.

Constantinou, M.C. and Tadjbakhsh, I.G. (1985, April) 'Hysteretic dampers in base isolation: random approach', Journal of Structural Engineering-ASCE, Vol. 111, No. 4, pp.705-721.

Doebling, S.W., Farrar, C.R., Prime, M.B. and Shevitz, D.W. (1996) Damage Identification and Health Monitoring of Structural and Mechanical Systems from Changes in their Vibration Characteristics: A Literature Review. Los Alamos National Laboratory, Report LA-13070-MS.

Doherty J.E. (1987) 'Non-destructive evaluation', in Kobayashi, A.S. (Ed): Handbook on Experimental Mechanics. Society for Experimental Mechanics, Bethel, CT, USA.

Hou, Z., Noori, M. and Amand, R. (2000) 'Wavelet-based approach for structural damage detection', Journal of Engineering Mechanics, Vol. 126, No. 7, pp.677-683.

Ifeachor, E.C. and Jervis, B.W. (1993) Digital Signal Processing: A Practical Approach, Addison-Wesley, Essex, UK.

Lim, M. and Lim, J. (2008) 'Visual measurement of pile movements for the foundation work using a high-speed line-scan camera', Journal of Pattern Recognition Society, Vol. 41, pp.2025-2033.

Loh, C-H., Lin, C-Y. and Huang, C-C. (2000) 'Time domain identification of frames under earthquake loadings', Journal of Engineering Mechanics, Vol. 126, No. 7, pp.693-703.

Sato, T. and Qi, K. (1998) 'Adaptive $\mathrm{H}_{\infty}$ filter: its application to structural identification', Journal of Engineering Mechanics, Vol. 124, No. 11, pp.1233-1240. 